Faculty of Sciences and Mathematics, University of Niš, Serbia

Available at: http://www.pmf.ni.ac.rs/filomat

Filomat 23:3 (2009), 103-113 DOI:10.2298/FIL0903103H

\title{
WIENER-TYPE INVARIANTS OF SOME GRAPH OPERATIONS*
}

\author{
S. Hossein-Zadeh, A. Hamzeh and A. R. Ashrafi
}

\begin{abstract}
Let $d(G, k)$ be the number of pairs of vertices of a graph $G$ that are at distance $k, \lambda$ a real number, and $W \lambda(G)=\sum_{k>1} d(G, k) k \lambda$. W $W(G)$ is called the Wiener-type invariant of $G$ associated to real number $\lambda$. In this paper, the Wiener-type invariants of some graph operations are computed. As immediate consequences, the formulae for reciprocal Wiener index, Harary index, hyperWiener index and Tratch-Stankevich-Zefirov index are calculated. Some upper and lower bounds are also presented.
\end{abstract}

\section{Introduction}

In this paper, we only consider simple graphs. Suppose $G$ is a simple graph. As usual, the distance between the vertices $u$ and $v$ of $G$ is denoted by $d_{G}(u, v)(d(u, v)$ for short). It is defined as the length of a minimum path connecting them. Let $d(G, k)$ be the number of pairs of vertices of a graph $G$ that are at distance $k, \lambda$ a real number, and $W \lambda(G)=\sum_{k \geq 1} d(G, k) k \lambda$. W $W(G)$ is called the Wiener-type invariant of $G$ associated to real number $\lambda$, see [3] for details. Note that $d(G, 0)$ and $d(G, 1)$ represent the number of vertices and edges, respectively. The case of $\lambda=1,-1$ and -2 are called the classical Wiener index [21], reciprocal Wiener index [16] and Harary index [2] and [17], respectively. The quantities $W W=$ $\frac{1}{2}\left[W_{1}+W_{2}\right]$ and $T S Z=\frac{1}{6} W_{3}+\frac{1}{2} W_{2}+\frac{1}{3} W_{1}$ are the so-called hyper-Wiener index and Tratch-Stankevich-Zefirov index [4].

The Cartesian product $G \times H$ of graphs $G$ and $H$ is a graph such that $V(G \times H)$ $=V(G) \times V(H)$, and any two vertices $(a, b)$ and $(u, v)$ are adjacent in $G \times H$ if and only if either $a=u$ and $b$ is adjacent with $v$, or $b=v$ and $a$ is adjacent with $u$, see [7] for details. The join $G=G_{1}+G_{2}$ of graphs $G_{1}$ and $G_{2}$ with disjoint vertex sets $V_{1}$ and $V_{2}$ and edge sets $E_{1}$ and $E_{2}$ is the graph union $G_{1} \cup G_{2}$ together with all the

* This research was supported in part by a grant from IPM (No. 87200113).

2000 Mathematics Subject Classifications. 05C12, 05C76.

Key words and Phrases. Wiener-type invariant, Cartesian product, join, symmetric difference, composition, disjunction.

Received: August 3, 2009

Communicated by Dragan Stevanović 
edges joining $V_{1}$ and $V_{2}$. The composition $G=G_{1}\left[G_{2}\right]$ of graphs $G_{1}$ and $G_{2}$ with disjoint vertex sets $V_{1}$ and $V_{2}$ and edge sets $E_{1}$ and $E_{2}$ is the graph with vertex set $V_{1} \times V_{2}$ and $u=\left(u_{1}, v_{1}\right)$ is adjacent with $v=\left(u_{2}, v_{2}\right)$ whenever $\left(u_{1}\right.$ is adjacent with $\left.u_{2}\right)$ or $\left(u_{1}=u_{2}\right.$ and $v_{1}$ is adjacent with $\left.v_{2}\right),[7$, p. 185]. The disjunction $G \vee H$ of graphs $G$ and $H$ is the graph with vertex set $V(G) \times V(H)$ and $\left(u_{1}, v_{1}\right)$ is adjacent with $\left(u_{2}, v_{2}\right)$ whenever $u_{1} u_{2} \in E(G)$ or $v_{1} v_{2} \in E(H)$. The symmetric difference $G \oplus H$ of two graphs $G$ and $H$ is the graph with vertex set $V(G) \times V(H)$ and $E(G \oplus H)=\left\{\left(u_{1}, u_{2}\right)\left(v_{1}, v_{2}\right) \mid u_{1} v_{1} \in E(G)\right.$ or $u_{2} v_{2} \in E(H)$ but not both $\}$.

The first Zagreb index was originally defined as $M_{1}(G)=\sum_{u \in V(G)} d(u)^{2}$. The first Zagreb index can be also expressed as a sum over edges of $G, M_{1}(G)=$ $\sum_{u v \in E(G)}[d(u)+d(v)]$, where $d(x)$ denotes the degree of vertex $x$ in $G$. We refer the reader to [18] for the proof of this fact and for more information on Zagreb index.

Throughout this paper, $C_{n}, P_{n}, K_{n}$ and $S_{n}$ denote the cycle, path, complete and star graphs on $n$ vertices. Also, $K_{m, n}$ denotes the complete bipartite graph. The complement of a graph $G$ is a graph $H$ on the same vertices such that two vertices of $H$ are adjacent if and only if they are not adjacent in $G$. The graph $H$ is usually denoted by $\bar{G}$. Our other notations are standard and taken mainly from $[1,6,20]$.

\section{Main Results}

In this section, some exact formulae for the Wiener-type invariants of the Cartesian product, composition, join, disjunction and symmetric difference of graphs are pre-

sented. We begin with the following crucial lemma related to distance properties of some graph operations.

Lemma 2.1. Let $G$ and $H$ be graphs. Then we have:

(a) $|V(G \times H)|=|V(G \vee H)|=|V(G[H])|=|V(G \oplus H)|=|V(G)| \cdot|V(H)|$, $|E(G \times H)|=|E(G)| \cdot|V(H)|+|V(G)| \cdot|E(H)|$, $|E(G+H)|=|E(G)|+|E(H)|+|V(G)| \cdot|V(H)|$, $|E(G[H])|=|E(G)| \cdot|V(H)|^{2}+|E(H)| \cdot|V(G)|$, $|E(G \vee H)|=|E(G)| \cdot|V(H)|^{2}+|E(H)| \cdot|V(G)|^{2}-2|E(G)| \cdot|E(H)|$, $|E(G \oplus H)|=|E(G)| \cdot|V(H)|^{2}+|E(H)| \cdot|V(G)|^{2}-4|E(G)| \cdot|E(H)|$.

(b) $G \times H$ is connected if and only if $G$ and $H$ are connected.

(c) If $(a, c)$ and $(b, d)$ are vertices of $G \times H$ then $d_{G \times H}((a, c),(b, d))=d_{G}(a, b)+$ $d_{H}(c, d)$.

(d) The Cartesian product, join, composition, disjunction and symmetric difference of graphs are associative and all of them are commutative except from composition. 


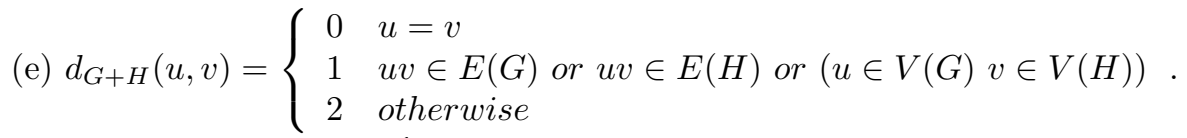

(f) $d_{G[H]}((a, b),(c, d))=\left\{\begin{array}{ll}d_{G}(a, c) & a \neq c \\ 0 & a=c \& b=d \\ 1 & a=c \& b d \in E(H) \\ 2 & a=c \& b d \notin E(H)\end{array}\right.$.

(g) $d_{G \vee H}((a, b),(c, d))=\left\{\begin{array}{ll}0 & a=c \& b=d \\ 1 & a c \in E(G) \text { or } b d \in E(H) \\ 2 & \text { otherwise }\end{array}\right.$.

(h) $d_{G \oplus H}((a, b),(c, d))=\left\{\begin{array}{ll}0 & a=c \& b=d \\ 1 & a c \in E(G) \text { or } b d \in E(H) \text { but not both . } \\ 2 & \text { otherwise }\end{array}\right.$.

Proof. The parts $(\mathrm{a}-\mathrm{e})$ are consequences of definitions and some well-known results of the book of Imrich and Klavžar, [7]. For the proof of $(\mathrm{f}-\mathrm{h})$ we refer to [11].

The Wiener index of the Cartesian product graphs was studied in [5, 19]. In [14], Klavžar, Rajapakse and Gutman computed the Szeged index of the Cartesian product graphs. The present authors, [8, 9, 10, 11, 12, 13, 22], computed some exact formulae for the hyper-Wiener, vertex PI, edge PI, the first Zagreb, the second Zagreb, the edge Wiener and the edge Szeged indices of some graph operations. The aim of this section is to continue this program for computing the Wiener-type invariants for five graph operations.

It is easy to see that $W_{\lambda}(G)=\sum_{k=1}^{l} d(G, k) k^{\lambda}$, where $l=\operatorname{diam}(G)$ denotes diameter of the graph $G$. Define $\alpha(k, r)=\sum_{i=1}^{k} i^{r}$. In the following simple lemma, we compute $W \lambda$ for five classes of known graphs.

Lemma 2.2. The following statements are hold:

1) $W \lambda\left(P_{n}\right)=n \alpha(n-1, \lambda)-\alpha(n-1, \lambda+1)$,

2) $W \lambda\left(K_{n}\right)=\left(\begin{array}{l}n \\ 2\end{array}\right)$,

3) $W \lambda\left(S_{n}\right)=(n-1)+\left(\begin{array}{c}n-1 \\ 2\end{array}\right) 2 \lambda$,

4) $W \lambda\left(K_{m, n}\right)=m n+2 \lambda\left(\left(\begin{array}{c}m \\ 2\end{array}\right)+\left(\begin{array}{c}n \\ 2\end{array}\right)\right)$,

5) $W \lambda\left(C_{n}\right)=\left\{\begin{array}{ll}n \alpha\left(\frac{n}{2}-1, \lambda\right)+\left(\frac{n}{2}\right) \lambda \frac{n}{2} & n \text { is even } \\ n \alpha\left(\frac{n-1}{2}, \lambda\right) & n \text { is odd }\end{array}\right.$.

Proposition 2.3. Let $G$ and $H$ be connected graphs. Then $W \lambda(G+H)=|E(G)|+$ $|E(H)|+|V(G)||V(H)|+2^{\lambda}(|E(\bar{G})|+|E(\bar{H})|)$.

Proof. By Lemma 2.1, we have:

$$
\begin{aligned}
W_{\lambda}(G+H) & =\sum_{k=1}^{2} d(G+H, k) k^{\lambda} \\
& =d(G+H, 1)+d(G+H, 2) 2^{\lambda} \\
& =|E(G)|+|E(H)|+|V(G)||V(H)|+2^{\lambda}(|E(\bar{G})|+|E(\bar{H})|),
\end{aligned}
$$


proving the result.

Corollary 2.4. The reciprocal Wiener index of $G+H$ is computed as follows:

$$
W_{-1}(G+H)=|E(G)|+|E(H)|+|V(G)||V(H)|+\frac{1}{2}(|E(\bar{G})|+|E(\bar{H})|) .
$$

Corollary 2.5. The Harary index of $G+H$ is computed as follows:

$$
W_{-2}(G+H)=|E(G)|+|E(H)|+|V(G)||V(H)|+\frac{1}{4}(|E(\bar{G})|+|E(\bar{H})|) .
$$

Corollary 2.6. The Tratch-Stankevich-Zefirov index of $G+H$ is computed as follows:

$$
T S Z(G+H)=|E(G)|+|E(H)|+|V(G)||V(H)|+4(|E(\bar{G})|+|E(\bar{H})|) .
$$

Corollary 2.7.(See [11, Theorem 2]) Let $G$ and $H$ be graphs. Then $W W(G+H)=$ $\frac{3}{2}|V(G)|^{2}+\frac{3}{2}|V(H)|^{2}-2|E(H)|-2|E(G)|-\frac{3}{2}|V(G)|-\frac{3}{2}|V(H)|+|V(G)||V(H)|$.

Corollary 2.8.(see [11, Corollary of Theorem 2].) Suppose $G_{1}, G_{2}, \ldots, G_{n}$ are graphs. Then

$$
W \lambda\left(G_{1}+G_{2}+\cdots+G_{n}\right)=(1-2 \lambda) \sum_{i=1}^{n}\left|E\left(G_{i}\right)\right|+\sum_{1 \leq i<j \leq n}\left|V\left(G_{i}\right)\right|\left|V\left(G_{j}\right)\right|+2 \lambda \sum_{i=1}^{n}\left(\begin{array}{c}
\left|V\left(G_{i}\right)\right| \\
2
\end{array}\right)
$$

In particular,

$$
W W\left(G_{1}+\ldots+G_{n}\right)=\sum_{i=1}^{n}\left(3\left(\begin{array}{c}
\left|V_{i}\right| \\
2
\end{array}\right)-2\left|E_{i}\right|\right)+\frac{1}{2} \sum_{i \neq j, i, j=1}^{n}\left|V_{i}\right|\left|V_{j}\right|
$$

and $W W(n G)=\frac{1}{2}\left(n^{2}+2 n\right)|V(G)|^{2}-2 n|E(G)|-\frac{3 n}{2}|V(G)|$, where $n G$ denotes the join of $n$ copy of $G$.

Proof. By a simple calculation,

$$
\begin{aligned}
& W \lambda\left(G_{1}+G_{2}+\cdots+G_{n}\right)=\sum_{i=1}^{n}\left|E\left(G_{i}\right)\right|+\sum_{1 \leq i<j \leq n}\left|V\left(G_{i}\right)\right|\left|V\left(G_{j}\right)\right|+2 \lambda\left[\sum_{i=1}^{n}\left|E\left(\bar{G}_{i}\right)\right|\right] \\
& =(1-2 \lambda) \sum_{i=1}^{n}\left|E\left(G_{i}\right)\right|+\sum_{1 \leq i<j \leq n}\left|V\left(G_{i}\right)\right|\left|V\left(G_{j}\right)\right|+2 \lambda \sum_{i=1}^{n}\left(\begin{array}{c}
\left|V\left(G_{i}\right)\right| \\
2
\end{array}\right),
\end{aligned}
$$

as desired.

Consider a complete $n$-partite graph $G=K_{m_{1}, m_{2}, \ldots, m_{n}}$ containing $v=|V(G)|$ vertices, Figure 1. By definition, in this graph the set of vertices can be partitioned into subsets $V_{1}, V_{2}, \ldots, V_{n}$ of $V$ such that for every i, $1 \leq i \leq n$, there is no edge between the vertices of $V_{i}$. 


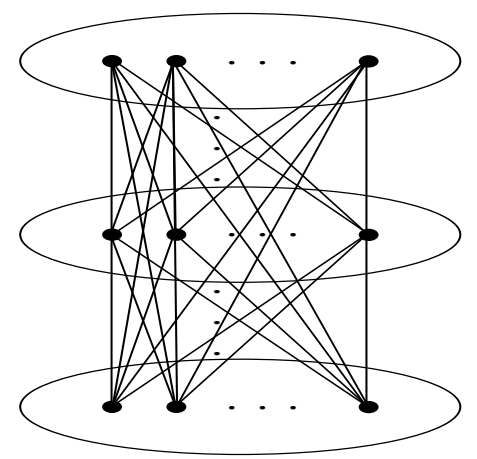

Figure 1. The Complete $n$-Partite Graph.

By the previous corollary, one can see that

$$
W_{\lambda}\left(K_{m_{1}, m_{2}, \ldots, m_{n}}\right)=\sum_{1 \leq i<j \leq n} m_{i} m_{j}+2^{\lambda} \sum_{i=1}^{n}\left(\begin{array}{c}
m_{i} \\
2
\end{array}\right) .
$$

Proposition 2.9. Let $G$ and $H$ be graphs. Then $W \lambda(G \vee H)=|E(G)||V(H)|^{2}$ $+|E(H)||V(G)|^{2}-2|E(G)||E(H)|+2 \lambda(|V(G)||E(\bar{H})|+|V(H)||E(\bar{G})|+$ $2|E(\bar{G})||E(\bar{H})|)$.

Proof. By Lemma 2.1 and definition of disjunction,

$$
\begin{aligned}
W_{\lambda}(G \vee H) & =\sum_{k=1}^{2} d(G \vee H, k) k^{\lambda} \\
& =d(G \vee H, 1)+d(G \vee H, 2) 2^{\lambda} \\
& =|E(G)||V(H)|^{2}+|E(H)||V(G)|^{2}-2|E(G)||E(H)| \\
& +2 \lambda(|V(G)||E(\bar{H})|+|V(H)||E(\bar{G})|+2|E(\bar{G})||E(\bar{H})|),
\end{aligned}
$$

proving the result.

Corollary 2.10. The reciprocal Wiener index of $G \vee H$ is computed as follows:

$$
\begin{aligned}
W_{-1}(G \vee H) & =|E(G)||V(H)|^{2}+|E(H)||V(G)|^{2}-2|E(G)||E(H)| \\
& +\frac{1}{2}(|V(G)||E(\bar{H})|+|V(H)||E(\bar{G})|+2|E(\bar{G})||E(\bar{H})|) .
\end{aligned}
$$

Corollary 2.11. The Harary index of $G \vee H$ is computed as follows:

$$
\begin{aligned}
W_{-2}(G \vee H) & =|E(G)||V(H)|^{2}+|E(H)||V(G)|^{2}-2|E(G)||E(H)| \\
& +\frac{1}{4}(|V(G)||E(\bar{H})|+|V(H)||E(\bar{G})|+2|E(\bar{G})||E(\bar{H})|)
\end{aligned}
$$


Corollary 2.12. The Tratch-Stankevich-Zefirov index of $G \vee H$ is computed as follows:

$$
\begin{aligned}
T S Z(G \vee H) & =|E(G)||V(H)|^{2}+|E(H)||V(G)|^{2} \\
& -2|E(G)||E(H)|+4(|V(G)||E(\bar{H})| \\
& +|V(H)||E(\bar{G})|+2|E(\bar{G})||E(\bar{H})|) .
\end{aligned}
$$

Corollary 2.13.(See [11, Theorem 4].) Let $G$ and $H$ be graphs. Then $W W(G \vee$ $H)=3(\underset{2}{|V(G)||V(H)|})+4|E(G)||E(H)|-2|V(H)|^{2}|E(G)|-2|V(G)|^{2}|E(H)|$.

Proposition 2.14. Let $G$ and $H$ be graphs. Then the Wiener-type invariant of the symmetric difference of $G$ and $H$ is: $W \lambda(G \oplus H)=2 \lambda(2|E(G)||E(H)|$ $+|V(H)||E(\bar{G})|+|V(G)||E(\bar{H})|+2|E(\bar{H})||E(\bar{G})|)+|E(G)||V(H)|^{2}+$ $|E(H)||V(G)|^{2}-4|E(G)||E(H)|$.

Proof. By Lemma 2.1 and definition of symmetric difference of two graphs, we have:

$$
\begin{aligned}
W_{\lambda}(G \oplus H) & =\sum_{k=1}^{2} d(G \oplus H, k) k^{\lambda} \\
& =d(G \oplus H, 1)+d(G \oplus H, 2) 2^{\lambda} \\
& =|E(G)||V(H)|^{2}+|E(H)||V(G)|^{2}-4|E(G)||E(H)| \\
& +2 \lambda(2|E(G)||E(H)|+|V(H)||E(\bar{G})|+|V(G)||E(\bar{H})| \\
& +2|E(\bar{H})||E(\bar{G})|),
\end{aligned}
$$

proving the result.

Corollary 2.15. The reciprocal Wiener index of $G \oplus H$ is computed as follows:

$$
\begin{aligned}
W_{-1}(G \oplus H) & =|E(G)||V(H)|^{2}+|E(H)||V(G)|^{2}-4|E(G)||E(H)| \\
& +\frac{1}{2}(2|E(G)||E(H)|+|V(H)||E(\bar{G})|+|V(G)||E(\bar{H})| \\
& +2|E(\bar{H})||E(\bar{G})|) .
\end{aligned}
$$

Corollary 2.16. The Harary index of $G \oplus H$ is computed as follows:

$$
\begin{aligned}
W_{-2}(G \oplus H) & =|E(G)||V(H)|^{2}+|E(H)||V(G)|^{2}-4|E(G)||E(H)| \\
& +\frac{1}{4}(2|E(G)||E(H)|+|V(H)||E(\bar{G})|+|V(G)||E(\bar{H})| \\
& +2|E(\bar{H})||E(\bar{G})|)
\end{aligned}
$$

Corollary 2.17. The Tratch-Stankevich-Zefirov index of $G \oplus H$ is computed as follows:

$$
\frac{1}{6} W_{3}(G \oplus H)+\frac{1}{2} W_{2}(G \oplus H)+\frac{1}{3} W_{1}(G \oplus H)=
$$




$$
\begin{array}{r}
=|E(G)||V(H)|^{2}+|E(H)||V(G)|^{2}-4|E(G)||E(H)|+4(2|E(G)||E(H)| \\
+|V(H)||E(\bar{G})|+|V(G)||E(\bar{H})|+2|E(\bar{H})||E(\bar{G})|)
\end{array}
$$

Corollary 2.18. (See $[11$, Theorem 5].) Let $G$ and $H$ be connected graphs. Then

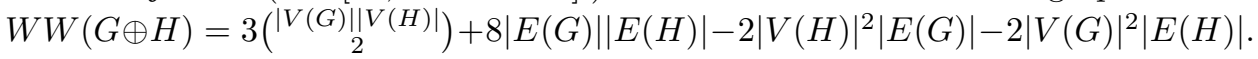

Proposition 2.19. Let $G$ and $H$ be graphs. Then $W_{\lambda}(G[H])=|V(H)|^{2} W_{\lambda}(G)+$ $|V(G)||E(H)|+2^{\lambda}|V(G)||E(\bar{H})|$.

Proof. Suppose $d_{1}(G[H], k)$ and $d_{2}(G[H], k)$ denote the number of 2 -subsets $\{(a, b),(x, y)\}$ such that $d_{G[H]}((a, b),(x, y))=k, a=x$ and $a \neq x$, respectively. In the first case, by Lemma $2.1 d_{G[H]}((a, b),(x, y)) \leq 2$ and so,

$$
\begin{aligned}
W_{\lambda}(G[H]) & =\sum_{k=1}^{2} d_{1}(G[H], k) k^{\lambda}+\sum_{k=1}^{d_{G}} d_{2}(G[H], k) k^{\lambda} \\
& =d_{1}(G[H], 1)+2^{\lambda} d_{1}(G[H], 2)+|V(H)|^{2} W_{\lambda}(G) \\
& =|V(G)||E(H)|+2^{\lambda}|V(G)||E(\bar{H})|+|V(H)|^{2} W_{\lambda}(G),
\end{aligned}
$$

proving the result.

Corollary 2.20. The reciprocal Wiener index of $G[H]$ is computed as follows:

$$
W_{-1}(G[H])=|V(H)|^{2} W_{-1}(G)+|V(G)||E(H)|+\frac{1}{2}|V(G)||E(\bar{H})| .
$$

Corollary 2.21. The Harary index of $G[H]$ is computed as follows:

$$
W_{-2}(G[H])=|V(H)|^{2} W_{-2}(G)+|V(G)||E(H)|+\frac{1}{4}|V(G)||E(\bar{H})| .
$$

Corollary 2.22. The Tratch-Stankevich-Zefirov index of $G[H]$ is computed as $T S Z(G[H])=|V(H)|^{2} T S Z(G)+|V(G)||E(H)|+4|V(G)||E(\bar{H})|$.

Corollary 2.23.(See [11, Theorem 3].) Let $G$ and $H$ be graphs and $G$ be connected. Then $W W(G[H])=|V(H)|^{2} W W(G)+\frac{|V(G)|}{2}\left(W W(2 H)-|V(H)|^{2}\right)$.

\section{Bounds on Wiener-Type Invariants of Graphs}

In this section some bounds on Wiener-type invariants of graphs are computed. We also find necessary and sufficient conditions for sharpness of these bounds.

Proposition 3.1. Let $G$ be a graph with exactly $n$ vertices and $m$ edges. If $\lambda \geq 0$ then

$2^{\lambda-1} M_{1}+m(1-2 \lambda)+l \lambda\left(\left(\begin{array}{c}n \\ 2\end{array}\right)-\frac{1}{2} M_{1}\right) \geq W_{\lambda}(G) \geq 2^{\lambda-1} M_{1}+m(1-2 \lambda)+3 \lambda\left(\left(\begin{array}{l}n \\ 2\end{array}\right)-\frac{1}{2} M_{1}\right)$ 
and if $\lambda \leq 0$ then

$2^{\lambda-1} M_{1}+m(1-2 \lambda)+l \lambda\left(\left(\begin{array}{l}n \\ 2\end{array}\right)-\frac{1}{2} M_{1}\right) \leq W_{\lambda}(G) \leq 2^{\lambda-1} M_{1}+m(1-2 \lambda)+3 \lambda\left(\left(\begin{array}{l}n \\ 2\end{array}\right)-\frac{1}{2} M_{1}\right)$.

Moreover, if $G$ is triangle and quadrangle free then the equalities hold if and only if $\operatorname{diam}(G)=3$.

Proof. Suppose $\lambda \geq 0$. By definition and [23, Eqs. 4 and 5],

$$
\begin{aligned}
W_{\lambda}(G) & =\sum_{k=1}^{l} d(G, k) k^{\lambda} \\
& =d(G, 1)+2 \lambda d(G, 2)+\sum_{k \geq 3} d(G, k) k \lambda \\
& \geq m+2 \lambda\left(\frac{1}{2} M_{1}-m\right)+3 \lambda \sum_{k \geq 3} d(G, k) \\
& =2^{\lambda-1} M_{1}+m(1-2 \lambda)+3 \lambda\left(\left(\begin{array}{l}
n \\
2
\end{array}\right)-\frac{1}{2} M_{1}\right),
\end{aligned}
$$

as desired. On the other hand,

$$
\begin{aligned}
W_{\lambda}(G) & =\sum_{k=1}^{l} d(G, k) k^{\lambda} \\
& =d(G, 1)+2 \lambda d(G, 2)+\sum_{k \geq 3} d(G, k) k \lambda \\
& \leq 2^{\lambda-1} M_{1}+m(1-2 \lambda)+l \lambda\left(\left(\begin{array}{l}
n \\
2
\end{array}\right)-\frac{1}{2} M_{1}\right) .
\end{aligned}
$$

The second part is an immediate consequence of above inequalities.

Proposition 3.2. Let $G$ and $H$ be graphs and $R$ and $S$ defined as follows:

$$
\begin{aligned}
R= & \frac{1}{2}|V(G)| M_{1}(H)(2 \lambda-d \lambda)+\frac{1}{2}|V(H)| M_{1}(G)(2 \lambda-d \lambda) \\
& +4|E(G)||E(H)|(2 \lambda-d \lambda)+d \lambda\left(\begin{array}{c}
|V(G)||V(H)| \\
2
\end{array}\right) \\
& +(1-2 \lambda)|V(G)||E(H)|+(1-2 \lambda)|V(H)||E(G)|, \\
S= & \frac{1}{2}|V(G)| M_{1}(H)(2 \lambda-3 \lambda)+\frac{1}{2}|V(H)| M_{1}(G)(2 \lambda-3 \lambda) \\
& +4|E(G)||E(H)|(2 \lambda-3 \lambda)+3 \lambda\left(\begin{array}{c}
|V(G)||V(H)| \\
2
\end{array}\right) \\
& +(1-2 \lambda)|V(G)||E(H)|+(1-2 \lambda)|V(H)||E(G)| .
\end{aligned}
$$


If $\lambda \geq 0$ then $R \geq W_{\lambda}(G \times H) \geq S$ and if $\lambda \leq 0$ then $S \geq W_{\lambda}(G \times H) \geq R$. Moreover, if $G \times H$ is triangle and quadrangle free then the equalities hold if and only if $\operatorname{diam}(G \times H)=3$.

Proof. Suppose $\lambda \geq 0$. By Lemma 2.1 and [8, Theorem 1], we have:

$$
\begin{aligned}
W_{\lambda}(G \times H) \geq & 2^{\lambda-1} M_{1}(G \times H)+3^{\lambda}\left(\left(\begin{array}{c}
n_{G \times H} \\
2
\end{array}\right)-\frac{1}{2} M_{1}(G \times H)\right) \\
& +(1-2 \lambda) m_{G \times H} \\
= & 2^{\lambda-1}\left(|V(G)| M_{1}(H)+|V(H)| M_{1}(G)+8|E(G)||E(H)|\right) \\
+ & 3 \lambda\left(\left(\begin{array}{c}
|V(G)||V(H)| \\
2
\end{array}\right)-\frac{1}{2}\left(|V(G)| M_{1}(H)+8|E(G)||E(H)|\right)\right. \\
+ & (1-2 \lambda)(|V(G)||E(H)|+|V(H)||E(G)|)-\frac{1}{2} 3 \lambda|V(H)| M_{1}(G) \\
= & \frac{1}{2}|V(G)| M_{1}(H)(2 \lambda-3 \lambda)+\frac{1}{2}|V(H)| M_{1}(G)(2 \lambda-3 \lambda) \\
+ & 4|E(G)||E(H)|(2 \lambda-3 \lambda)+3 \lambda\left(\begin{array}{c}
|V(G)||V(H)| \\
2
\end{array}\right) \\
+ & (1-2 \lambda)|V(G)||E(H)|+(1-2 \lambda)|V(H)||E(G)| .
\end{aligned}
$$

Let $d$ denote the diameter of $G \times H$. Then

$$
\begin{aligned}
W_{\lambda}(G \times H) & \leq \frac{1}{2}|V(G)| M_{1}(H)(2 \lambda-d \lambda)+\frac{1}{2}|V(H)| M_{1}(G)(2 \lambda-d \lambda) \\
& +4|E(G)||E(H)|(2 \lambda-d \lambda)+d \lambda\left(\begin{array}{c}
|V(G)||V(H)| \\
2
\end{array}\right) \\
& +(1-2 \lambda)|V(G)||E(H)|+(1-2 \lambda)|V(H)||E(G)| .
\end{aligned}
$$

We now assume that $\lambda \leq 0$. Then,

$$
\begin{aligned}
W_{\lambda}(G \times H) & \leq \frac{1}{2}|V(G)| M_{1}(H)\left(2 \lambda-3^{\lambda}\right)+\frac{1}{2}|V(H)| M_{1}(G)\left(2 \lambda-3^{\lambda}\right) \\
& +4|E(G)||E(H)|\left(2 \lambda-3^{\lambda}\right)+3^{\lambda}\left(\begin{array}{c}
|V(G)||V(H)| \\
2
\end{array}\right) \\
& +(1-2 \lambda)|V(G)||E(H)|+(1-2 \lambda)|V(H)||E(G)|
\end{aligned}
$$

and,

$$
\begin{aligned}
W_{\lambda}(G \times H) & \geq \frac{1}{2}|V(G)| M_{1}(H)\left(2 \lambda-d^{\lambda}\right)+\frac{1}{2}|V(H)| M_{1}(G)\left(2 \lambda-d^{\lambda}\right) \\
& +4|E(G)||E(H)|\left(2 \lambda-d^{\lambda}\right)+d^{\lambda}\left(\begin{array}{c}
|V(G)||V(H)| \\
2
\end{array}\right) \\
& +(1-2 \lambda)|V(G)||E(H)|+(1-2 \lambda)|V(H)||E(G)|,
\end{aligned}
$$

which completes our argument.

Acknowledgement. We are greatly indebted to the referees for their corrections and helpful remarks. 


\section{References}

[1] M. V. Diudea, I. Gutman and L. Jantschi, Molecular Topology, Huntington, NY, 2001.

[2] M. V. Diudea, Indices of reciprocal properties or Harary indices, J. Chem. Inf. Comput. Sci., 37 (1997), 292-299.

[3] I. Gutman, A property of the Wiener number and its modifications, Indian J. Chem., 36A (1997), 128-132.

[4] I. Gutman, A. A. Dobrynin, S. Klavžar and L. Pavlović, Wiener-type invariants of trees and their relation, Bull. Inst. Combin. Appl., 40 (2004), 23-30.

[5] A. Graovac and T. Pisanski, On the Wiener index of a graph, J. Math. Chem. 8(1991), 53-62.

[6] F. Harary, Graph Theory, AddisonWesley, Reading, MA, 1969.

[7] W. Imrich and S. Klavžar, Product graphs: structure and recognition, John Wiley \& Sons, New York, USA, 2000.

[8] M. H. Khalifeh, H. Yousefi-Azari and A. R. Ashrafi, The first and second Zagreb indices of some graph operations, Discrete Appl. Math., 157(4) (2009), 804-811.

[9] M. H. Khalifeh, H. Yousefi-Azari, A. R. Ashrafi, and S. G. Wagner, Some new results on distance-based graph invariants, European J. Combin., 30(5) (2009), $1149-1163$.

[10] M. H. Khalifeh, H. Yousefi-Azari, A. R. Ashrafi, A matrix method for computing Szeged and vertex PI indices of join and composition of graphs, Linear Algebra Appl., 429(11-12) (2008), 2702-2709.

[11] M. H. Khalifeh, H. Yousefi-Azari and A. R. Ashrafi, The hyper-Wiener index of graph operations, Comput. Math. Appl., 56(5) (2008), 1402-1407.

[12] M. H. Khalifeh, H. Yousefi-Azari and A. R. Ashrafi, Vertex and edge PI indices of Cartesian product graphs, Discrete Appl. Math., 156(10) (2008), 1780-1789.

[13] M. H. Khalifeh, H. Yousefi-Azari, A. R. Ashrafi, I. Gutman, The edge szeged index of product graphs, Croat. Chem. Acta, 81(2) (2008), 277-281.

[14] S. Klavžar, A. Rajapakse and I. Gutman, The Szeged and the Wiener index of graphs, Appl. Math. Lett., 9(1996), 45-49.

[15] S. Klavžar, On the PI index: PI-partitions and Cartesian product graphs, MATCH Commun. Math. Comput. Chem., 57(3) (2007), 573-586.

[16] S. Klavžar and I. Gutman, A theorem on Wiener-type invariants for isometric subgraphs of hypercubes, Appl. Math. Lett., 19 (2006), 1129-1133. 
[17] B. Lučić, A. Miličević, S. Nikolić and N. Trinajstić, Harary index - twelve years later, Croat. Chem. Acta, 75(4) (2002), 847-868.

[18] S. Nikolić, G. Kovačević, A. Miličević, N. Trinajstić, The Zagreb Indices 30 Years After, Croat. Chem. Acta, 76(2003), 113-124.

[19] B. E. Sagan, Y.-N. Yeh and P. Zhang, The Wiener polynomial of a graph, Int. J. Quant. Chem., 60 (5)(1996), 959-969.

[20] N. Trinajstić, Chemical Graph Theory, CRC Press, Boca Raton, FL. 1992.

[21] H. Wiener, Structural determination of the paraffin boiling points, J. Am. Chem. Soc., 69(1947), 17-20.

[22] H. Yousefi-Azari, B. Manoochehrian and A. R. Ashrafi, The PI index of product graphs, Appl. Math. Lett., 21(6) (2008), 624-627.

[23] B. Zhou and I. Gutman, Relations between Wiener, hyper-Wiener and Zagreb indices, Chem. Phys. Lett., 394 (2004), 93-95.

Department of Mathematics, Faculty of Science, University of Kashan, Kashan 87317-51167, Iran

School of Mathematics, Institute for Research in Fundamental Sciences (IPM), P.O.

Box: 19395-5746, Tehran, Iran

E-mail: ashrafi@kashanu.ac.ir 